\title{
Expression of BMP2-Hydrophobin fusion protein in the tobacco plant and molecular dynamic evaluation of its simulated model
}

\author{
Pouya Rahimifard Hamedani ${ }^{1} \cdot$ Mahmood Solouki $^{1} \cdot$ Parastoo Ehsani $^{2} \cdot$ Abbasali Emamjomeh $^{1} \cdot$ Hamideh Ofoghi $^{3}$
}

Received: 23 January 2021 / Revised: 14 May 2021 / Accepted: 2 June 2021 / Published online: 11 June 2021

(c) Korean Society for Plant Biotechnology 2021

\begin{abstract}
Plants are one of the ideal models for therapeutic protein production, however the recombinant protein purification problems in them must be overcome. Bone Morphogenetic Protein2 (BMP2) is employed for the restoration and construction of bone tissues. Hydrophobin is a fungal based protein with high hydrophobic characteristics. Due to this specificity, it is suitable for the purification of chimer protein from complex solutions when is fused to a protein utilizing an aqueous two-phase (A2P) technique. The plant optimized mature human BMP2 gene was designed and evaluated by in silico method. This process involves simulating molecular dynamics using the RMSD, RMSF and Gyration radius indexes. The synthesized Hyd-BMP2 gene was cloned into a pTRAkc-ERH plasmid and Transferred into Agrobacterium (Gv3101). The Nicotiana benthamiana plant leaves were co-agroinfiltrated with HA-Hyd-BMP2 and P19-pCambia1304 containing silencing suppressor. After purification of plant extract utilizing the A2P method, the sample was subjected to SDS-PAGE and Western-blot. By in silico study, the simulated fusion protein profitably shows reasonable protein compactness and the effect of amino acid substitution on protein-protein interaction is not remarkable. Western-blotting using anti HA tag has shown that the A2P technique partially purified the two $22 \mathrm{kDa}$ and $44 \mathrm{kDa}$ forms of Hydrophobin-BMP2. These results confirmed the presence of monomer and dimer forms of Hydrophobin-BMP2 proteins. Moreover, the expression level of the protein using P19 silencing suppressor increased six times and to $0.018 \%$ as shown by ELISA. This study presents a fast and easy technique for the purification of transient expressed pharmaceutical proteins from plants.
\end{abstract}

Keywords Bone morphogenetic protein- $2 \cdot$ Hydrophobin $\cdot$ Molecular dynamics

\section{Introduction}

Regarding high demand for medical proteins, the production of recombinant pharmaceutical proteins has experimented in various prokaryotic and eukaryotic hosts. Plants are suitable systems as they have the posttranslational eukaryotic system, cheap and easy to scale up. Therefore, it is an ideal model for the expression of medical and diagnostic human

Mahmood Solouki

mahmood.solouki@gmail.com

Parastoo Ehsani

p_ehsani@pasteur.ac.ir

1 Department of Plant Breeding and Biotechnology (PBB), University of Zabol, Zabol, Iran

2 Department of Molecular Biology, Pasteur Institute of Iran, Tehran, Iran

3 Department of Biotechnology, Iranian Research Organization for Science and Technology, Tehran, Iran origin proteins (Merlin et al. 2014). Moreover, one of the most important capabilities of plant cells is induction o correct folding and assembly of the expressed protein which is crucial for the production of antibodies to recognize their related antigens (Khalesi et al. 2012) and pharmaceutical proteins to bound to their receptors such as growth regulatory proteins (Tiwari et al. 2009).

Bone morphogenetic protein-2 (BMP2) classified as the transforming growth factor-beta (TGF-b) superfamily that is needed in the development of many tissues such as the establishment of the fundamental embryonic body plan (Inai et al. 2008). The presence of cysteine amino acid in the BMP2 structure allows dimers to form from monomers via disulfide bonds (Zhang et al. 2012) and creates a hydrophobic core between the monomers (Weber et al. 2007). BMP2 is especially important in the pharmaceutical and therapeutic applications for the restoration and construction of bone tissue (Gamer et al. 2018) and treatment of chronic kidney disease (Miyata et al. 2018). It has great potential for genetic 
engineering researches (Zhang et al. 2010). The recombinant human BMP2 has been produced with several expression systems as active protein in mammalian cell cultures (Israel et al. 1992) or in the formation of inclusion bodies in the prokaryotic systems such as Escherichia coli (Vallejo et al. 2002). However, the low amounts of BMP2 expression are one of their restriction parameters (Tsuda et al. 2003).

Hydrophobins are found in some fungus systems and because of their hydrophobicity, they could be a solution for purifying low-level expressed and unstable fused recombinant protein. (Joensuu et al. 2010). Some eukaryotic hosts like plant, yeast, fungi and prokaryotic host like Escherichia Coli have been utilized for the production of hydrophobin as fused protein to use for bioseparation, protein purification, drug solubility, biosensor designing, and tissue engineering (Berger and Sallada 2019).

Non-ionic surfactants such as X114 are molecules that have two parts of water-compatible and repellent. The hydrophilic part of a fusion protein containing hydrophobicpolar domains interact with the aqueous phase and could be purified applying two phase system containing non-ionic surfactants (Sivars and Tjerneld 2000). Moreover, purification using the aqueous two-phase (A2P) system performed at $25-30{ }^{\circ} \mathrm{C}$ is suitable for in vitro studies (Joensuu et al. 2012); (Holmberg 2003). Therefore, in the present study the sequence of Hydrophobin BMP2 fusion protein was prepared and analyzed by in silico programs, then the construct gene was cloned and expressed using transient expression system. The Hydrophobin-BMP2 fusion protein was purified by $\mathrm{A} 2 \mathrm{P}$ and characterized.

\section{Materials and methods}

\section{Hydrophobin-BMP2 fusion protein design}

The coding sequences of BMP2 protein (AC-1REW-A) (Kirsch et al. 2000) and hydrophobin protein (AC-2FZ6-A) (Hakanpää et al. 2006) were taken from NCBI. The cassette designed in following order: NCoI restriction enzyme site, the Kozak sequence for plant, the DNA coding sequence of HA tag (YPYDVPDYA), hydrophobin gene, the DNA coding sequence of an Entrokinase recognition site (AspAsp-Asp-Asp-Lys), the BMP2 gene and finally BamHI Site. Then it was optimized using plant codon adaptation index and synthesized by Biomatik Company (Ontario, Canada) for expression in tobacco plants.

\section{In silico analysis}

The protein sequence of the Hydrophobin (PDB accession number: 16882996) and BMP2 (PDB accession number: 24311780) were taken from Protein Data Bank. GROMACS (version 5.1.2) software was used for in Silico analysis. Molecular dynamics simulation was done for protein structure within $20 \mathrm{~ns}$ (Bedrov et al. 2019). Sodium and potassium ions were used to neutralize the system (Kohagen et al. 2016). Simulations were performed for periodic boundary conditions (Yeh and Hummer 2004). Van der Waals forces were applied with Cut-off of $1.2 \AA$ (MacKerell and Karplus 1991). Particle-Mesh Ewald method also was used with a cut-off of $1.2 \AA$. (Nocito and Beran 2018). Frequency number were set on 10 to update neighbor list. Package of Gromacs protonation state was used to calculate the overall system load. Each simulation was conducted in four stages (Lemkul et al. 2015). The entire system was minimized by the steepest descent in the first stage (Bedrov et al. 2019). The movement of heavy atoms was limited by a constant force of $1000 \mathrm{~kJ} / \mathrm{mol} \mathrm{nm}$ in the second stage (equilibration) (Lemkul et al. 2016). Those operations were done respectively in the ensemble of NVT and NPT for 100 picoseconds (Childers and Daggett 2018). The system temperature was set at $25^{\circ} \mathrm{C}$ (to help Maxwell-Boltzmann distribution) and the pressure was set to one atmosphere. (Bhargava and Balasubramanian 2005). Temperature coupling and pressure coupling options, respectively, were set on 0.1 and 2 picoseconds (Ren and Ponder 2004). During equilibration for thermostat and barostat, V-rescale algorithm and ParrinelloRahman was used respectively. All links were considered by LINCS algorithm. In the last $20 \mathrm{ns,} \mathrm{molecular} \mathrm{dynamics}$ simulation was performed under the NPT ensemble while the temperature and pressure of Nose-Hoover thermostat and Parrinello-Rahman barostat were constant.

\section{Gene construction and cloning}

The PTRAkc-ERH expression vector was kindly provided by prof. Schillberg (Hanover Germany) and used for cloning and transformation of Agrobacterium (Gv3101:Pmp90RK strain). Nicotiana benthamiana was used as a plant host for co-agroinfiltration experiments. The synthesized gene (Hyd-BMP2) was digested by NcoI and BamHI and cloned into the PTRAkc ERH vector. Then it transferred into the $E$. Coli by heat shock technique (Sambrook 2001). Following confirmation of the presence of Hyd- BMP2, the expression vector was purified and transferred into Agrobacterium tumefaciens by the rapid-melting-freezing method (Chen et al. 1994). The presence of the construct was determined by two designed primers of F-Hyd (5'TAA TCCATGGCA TATCCATATGATG3') and R-BMP (5'TAATGGATCCTC ATCTACATCCG 3').

\section{Co-agroinfiltration and protein partial purification}

The desirable vector was transformed into tobacco leaves by the agroinjection method using the needle-less syringe (Kapila 
et al. 1997). For increasing the expression, co-agroinfiltration (while the $\mathrm{OD}_{600}$ was 0.3) was done with agrobacteria LBA4404 carrying P19-pCambia1304 (P19 gene coding for a silencing suppressor from Tomato Bushy Stunt Virus) vector that was already constructed in the lab (Mohammadzadeh et al. 2014). The leaves were harvested on the 6th day after the injection, extracted with PBS, and subjected to A2P (Joensuu et al. 2012). Briefly, $1 \mathrm{ml}$ of crude extract of the agroinjected leaves mixed with $40 \mathrm{mg}$ of triton $\mathrm{X}-114$, then it was placed at $24^{\circ} \mathrm{C}$ for $5 \mathrm{~min}$ so the two phases separated. The lower phase was taken and $400 \mu \mathrm{l}$ of isobutanol (Sigma-Aldrich) was added, vortexed, and placed at $24{ }^{\circ} \mathrm{C}$ for $5 \mathrm{~min}$ again. Following the separation of two phases, the lower phase was saved at $-70^{\circ} \mathrm{C}$ for further analysis. (Kurppa et al. 2018).

\section{Protein characterization}

The control and co-agroinfiltrated leaves were extracted using PBS, containing $2 \mathrm{mM}$ PMSF with mortar and pestle. The different phases of plant extract from the A2P experiment were examined by SDS-PAGE on $10 \%$ (w/v) polyacrylamide gels. Western blotting was done by transferring proteins from the gel into a nitrocellulose membrane. Following blocking with $1 \%$ skimmed milk, the membrane was probed with monoclonal rabbit Anti-HA IgG (1:2000 Sigma-Aldrich; H6908). The results were detected using the anti-rabbit antibody HRP conjugated (1:4000 Sigma-Aldrich; AP187P) and revealed by DAB.

\section{ELISA}

The concentration of BMP2 and the P19 protein effect on the amount of expressed recombinant protein was evaluated by the ELISA. The ELISA plates were coated with $100 \mu \mathrm{l}$ of monoclonal anti-HA antibody $(5 \mu \mathrm{g} / \mathrm{ml})$ (Abcam, UK) in carbonate buffer for $16 \mathrm{~h}$. The plates were washed three times with PBS and $100 \mu \mathrm{l}$ of plant protein extract $(50 \mu \mathrm{g})$ with and without p19 were loaded in each well incubated for $2 \mathrm{~h}$ at $37^{\circ} \mathrm{C}$ and washed. Then a polyclonal rabbit AntiBMP2-HRP (Abcam, UK) antibody (1/4000) was used as an antigen detector and TMB was used as a substrate. The plates were placed in the ELISA plate reader while the absorbance of each sample is read at $450 \mathrm{~nm}$ to determine the amount of recombinant protein in total soluble protein (TSP).

\section{Result}

\section{Molecular dynamic analysis of Hydrophobin-BMP2 protein for prediction of its behavior}

Following designing the construct, the behavior of the protein was evaluated using bioinformatics tools. The RMSD
(Root Mean Square Deviation) between the structures created to the dimension of time is a common selection criterion to ensure the sustainability of molecular dynamics simulation (Fig. 1a). RMSF (Root Mean Square Fluctuation) was considered to study the movement and flexibility of the structure. This chart shows the fluctuation rate is per residues. As they could be seen, there are more volatility in some areas: residues 17 to 19,83 to 85,88 to 90 and 96 to 98 (Fig. 1b). The radius of gyration ( $\mathrm{Rg})$ is an indicator of protein structure compactness as if it shows the change in the protein structure compactness during a simulation and it is considered as a protein stability factor. Due to the fact that the gyration radius on average throughout the simulation shows low oscillations (1.8-1.9 nm) (Fig. 1c), the hydrophobin-BMP2 protein has stable compactness and form in the simulation process.

\section{Confirmation of agrobacteria transformation and transfer of Hyd-BMP2 construct to tobacco leaves}

The plant optimized expression construct (622 bp) contains mature hBMP2 gene (363 bp) with "Kozak" sequence for effective and better translations, Hydrophobin gene (204 bp), HA-tag, and an enterokinase cleavage site is shown in Fig. 2. The synthesized (Hyd- BMP2) construct (660 bp) was cloned into the PTRAkc and the presence of the construct in agrobacterium was tested by colony PCR on isolated clones. The amplification of the Hyd-BMP2 gene $660 \mathrm{bp}$ band using the primers F-Hyd and R-BMP2 was confirmed for the clones grown on selective media (Fig. 3).

The leaves of Nicotiana benthamiana were co-agroinfiltrated with Agrobacterium tumefaciens containing HydBMP2- pTRAkc vector and P19-pCambia1304 (Fig. 4). The plants used in this study (Fig. 4a), the agroinjected leaves following 5 min (Fig. 4b, c) and 6 days (Fig. 4d) post agroinjection. The dashed circles are the syringe injection area.

\section{Hyd-BMP2 Protein purified by A2P, characterized and level of expression determined}

The purification steps of the A2P technique for Hydrophobin-BMP2 fusion protein and control leaves are shown in Fig. 5. Addition of Triton X114 to TSP (Fig. 5a) produces two phases at step one. Then addition of Isobutanol to the lower phase of previous step in step two shows two phases (Fig. 5b) and the lower phases were tested for Hyd-BMP2 protein.

The four A2P preparations of TSP of leaves infiltrated with agrobacteria contain Hyd-BMP2 and P19 (Fig. 6a; 1, 2, 3 , the lower phase of step two), only control vector (Fig. 6a; 4 the lower phase of step two) and control vector (Fig. 6a; 5 , upper phase of step two) were analyzed by SDS-PAGE 

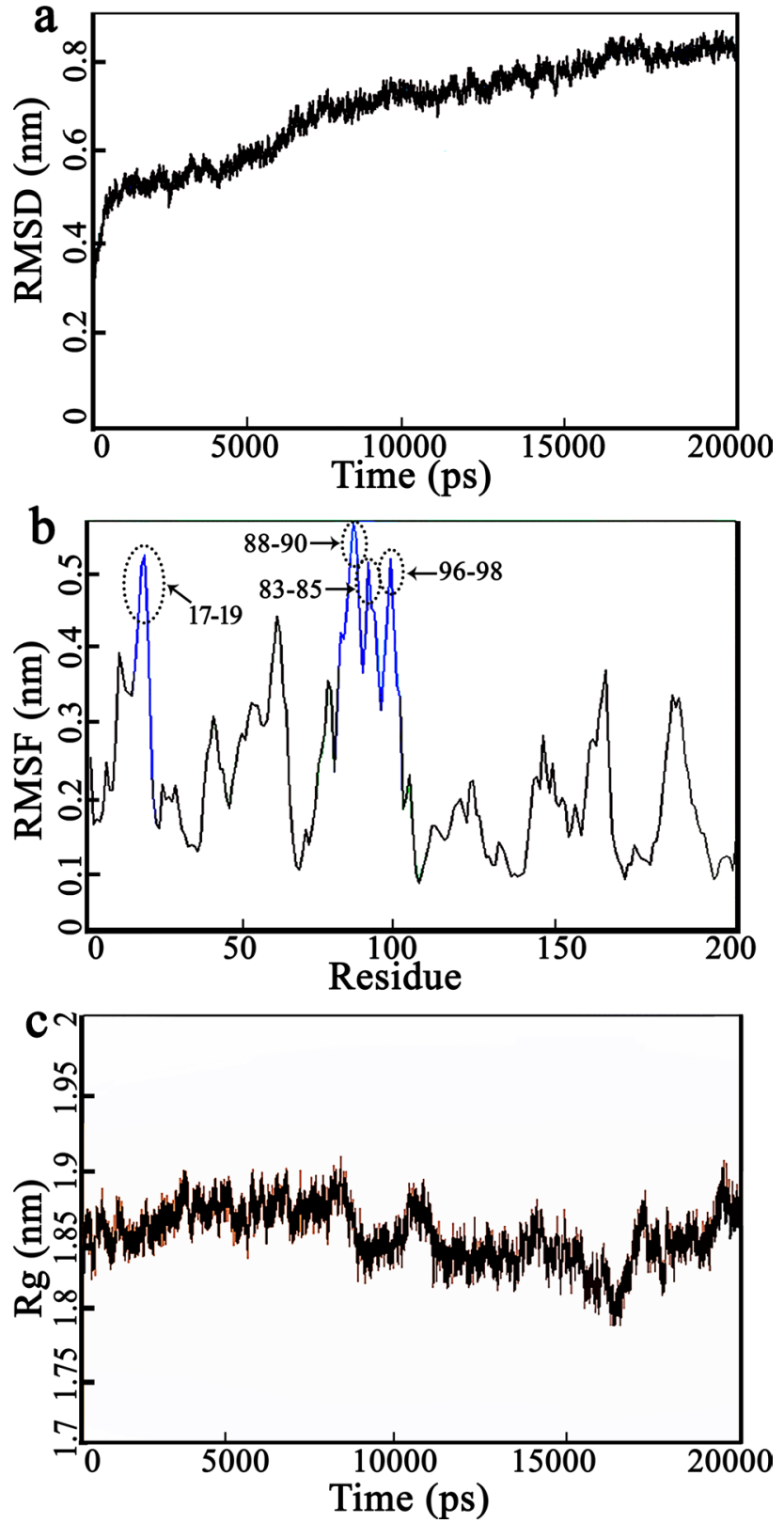

Fig. 1 The in silico analysis of Hyd-BMP2. a According to the RMSD diagram, structure is reached to relative stability about first 15,000 picoseconds. More stability was observed if the simulation continued for a longer period. There is acceptable stability in the range of $0.8 \mathrm{~nm}$. b RMS fluctuation shows the rate of fluctuation per residue in the range of $0.5 \mathrm{~nm}$ indicate. The four maximum areas (residues 17 to 19,83 to 85,88 to 90 and 96 to 98 ) that show the highest volatility are distinguished by dashed ovals. $\mathbf{c}$ The rate of changes in the radius of gyration in the range of 20,000 picoseconds

and Western blotting (Fig. 6b). SDS PAGE have shown a non-specific band at $24 \mathrm{kDa}$ that could be seen in all lower phase preparations of step two including control sample. However, Western blot shows approximately $22 \mathrm{kDa}$ (for monomer) and $44 \mathrm{kDa}$ (for dimer) bands in Hyd-BMP2 and

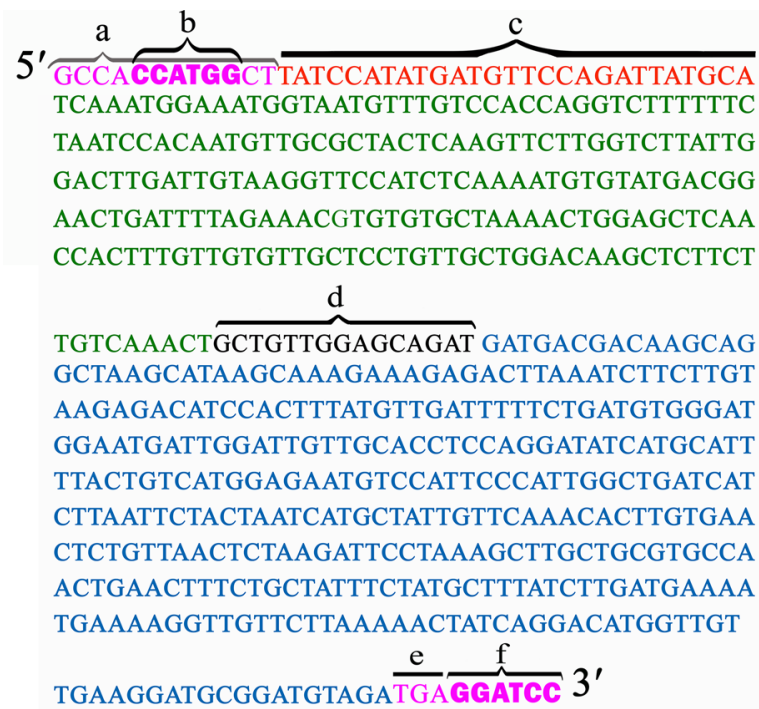

Fig. 2 The designed cassette of Hyd-BMP2, Hydrophobin, and BMP2 gene are respectively the in the green and blue area. a the Kozak sequence corresponding to the expression of the gene in the plant. b NCoI bound restriction enzyme position. $\mathbf{c}$ Followed by HA tag d enterokinase site. e Stop codon. $\mathbf{f}$ BamHI restriction site

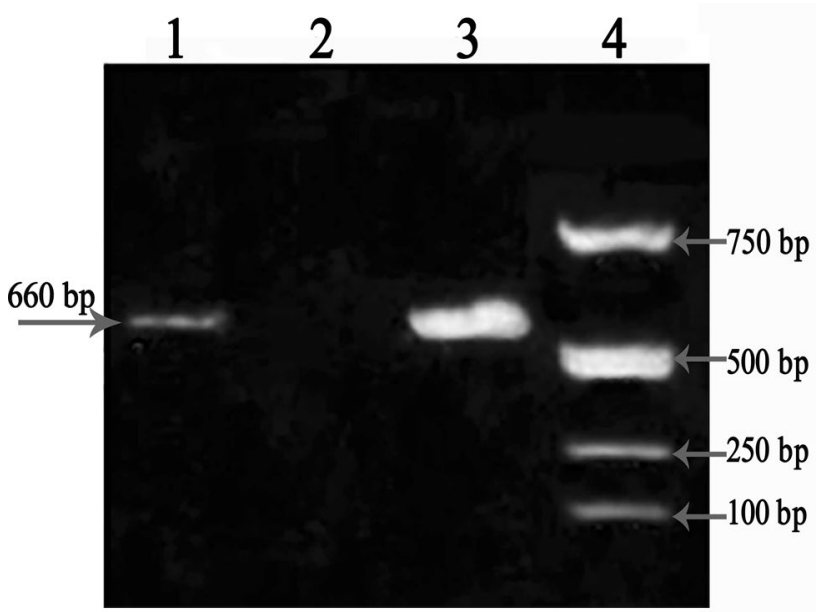

Fig. 3 Verification of presence of construct in Agrobacterium by Colony-PCR. a Plasmid containing cassette positive control. b Negative control. c Transformed agrobacteria. d 1 kb Marker

not in control, indicating that the Hydrophobin-BMP2 fusion protein had been expressed (Fig. 6b; 1, 2, 3).

The amount of the expression of the Hyd-BMP2 in the presence or absence of the P19 silencing suppressor construct was determined using ELISA method. The results of one-way ANOVA showed that there was a significant difference between the groups $(P<0.05)$. The amount of $30 \mathrm{ng} / \mathrm{g}$ $(0.003 \%)$ and $180 \mathrm{ng} / \mathrm{g}(0.018 \%)$ recombinant protein in TSP of fresh leaf was calculated for no P19 (Fig. 7b) and with P19 constructs (Fig. 7c), respectively. 


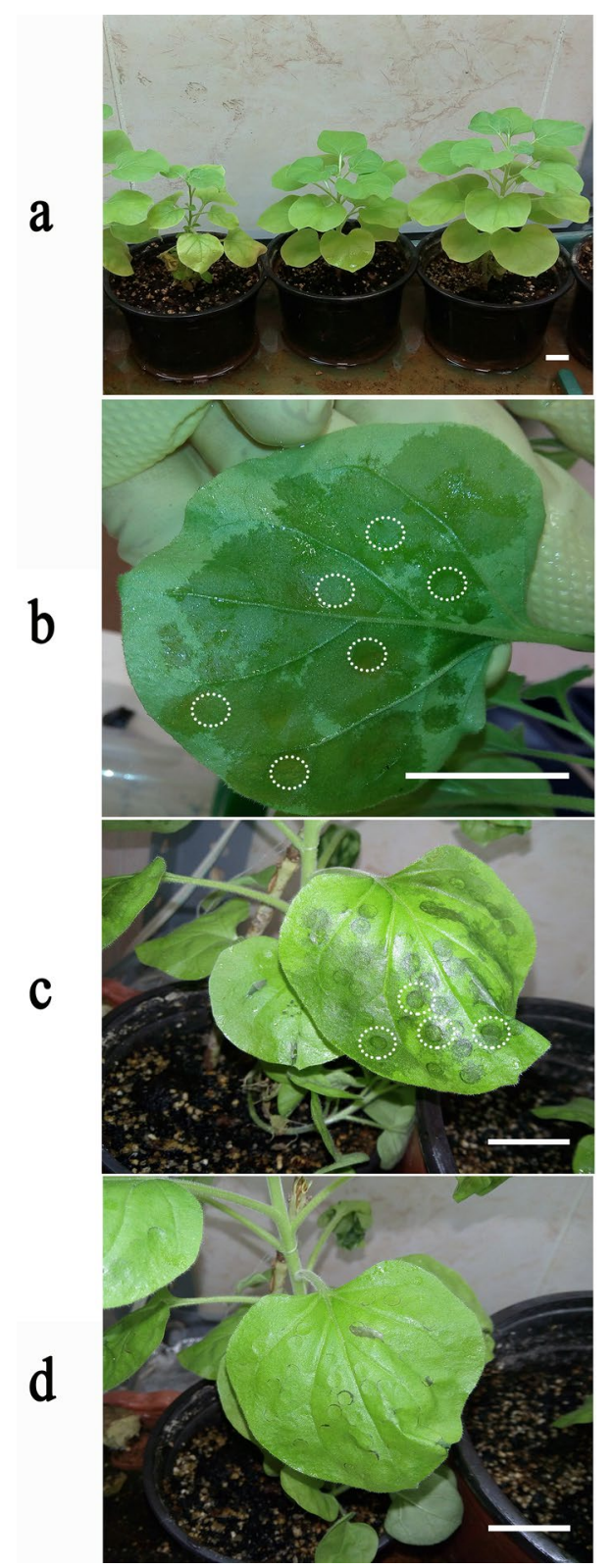

Fig. 4 Transfer of Agrobacterium containing hydrophobin-BMP2 construct by agroinjection method to the interstitial space of plant leaves. a The three selected plants after 6 weeks from seed germination. $\mathbf{b}$ The marked areas show the bacterial suspension infiltrated in the leaf following injection $\mathbf{c}$ Top of the leaves following injection. $\mathbf{d}$ The same leaves after 4 days of injection. Scale bar, $30 \mathrm{~mm}$

\section{Discussion}

BMP2 is needed for bone-related medical therapeutic applications and tissue engineering research. Different biotechnological systems produced BMP2 including transgenic plants (Gao et al. 2006; Dehghani et al. 2018) However, plant transient expression system has its special benefits, such as low cost, convenient to work and fast producer of high amount of bioactive protein compared to transgenic

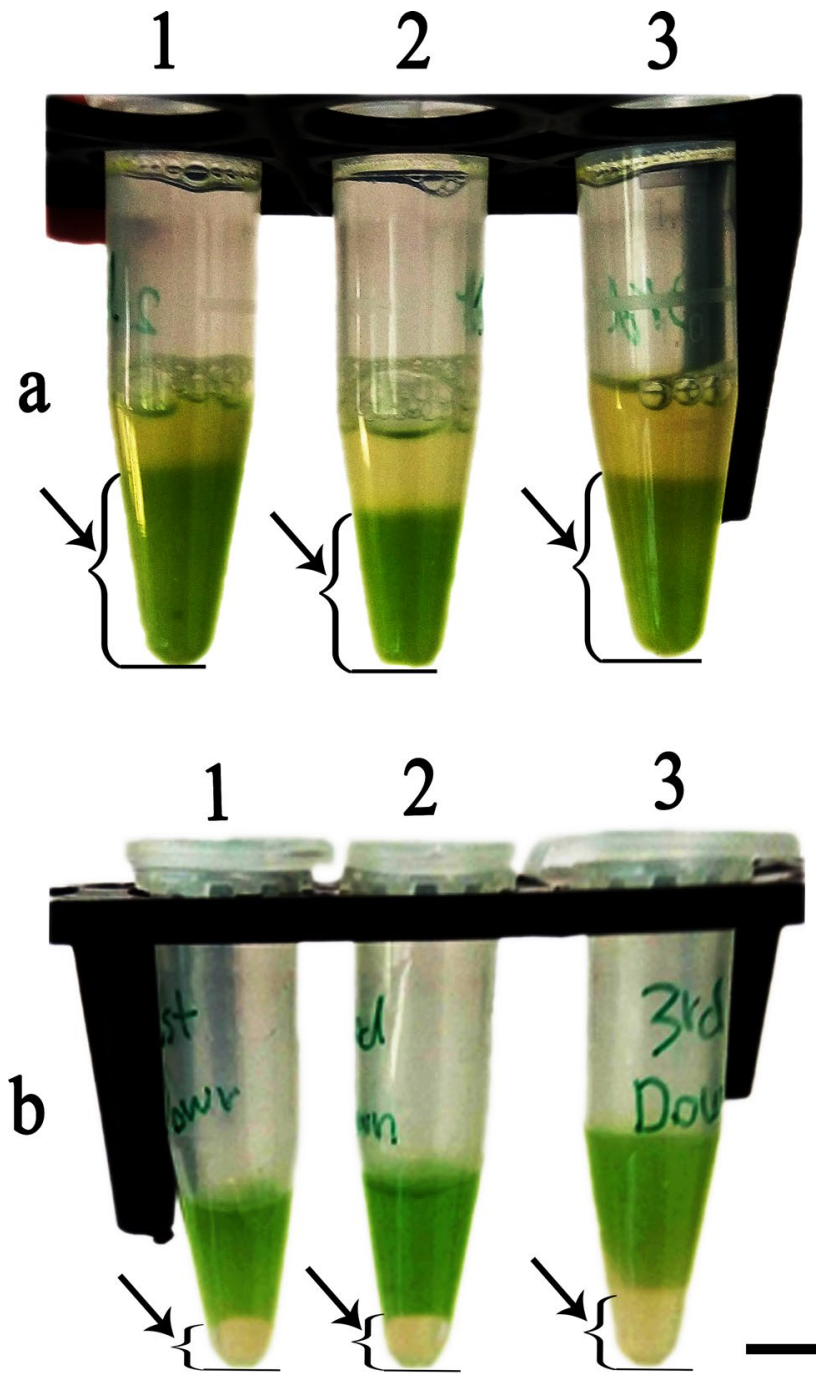

Fig. 5 Purification of the Hydrophobin-BMP2 fusion protein by A2P method. Vials 1,2 containing TSP of Hyd-BMP2 agroinjected leaves respectively and vial 3 is TSP of control agroinjected leaves. a In step one of the A2P, following adding the X114. b In step two of A2P following adding isobutanol. The marked area are the parts that are used for the next steps. Scale bar, $5 \mathrm{~mm}$

plant. Due to presence of different classes of impurities in plant expressed product, downstream purification is a time consuming process and may results in major loss of pharmaceutical recombinant protein. This is first study on plant transient expression of BMP2 fusion protein and the goal of this research is fast and facile purifying of expressed BMP2 fused to hydrophobin from tobacco leaves and increasing the expression level using P19 silencing suppressor.

In silico studies confirmed that the designed construct and the sequence of its components are suitable for the production of hyd-BMP2 protein and do not affect the protein stability as it was done for TGF $\alpha$ L3-SEB human fusion protein (as a new antitumor agent) (Imani-Fooladi et al. 2014). Moreover, Shamriz et al. has shown that the designed 

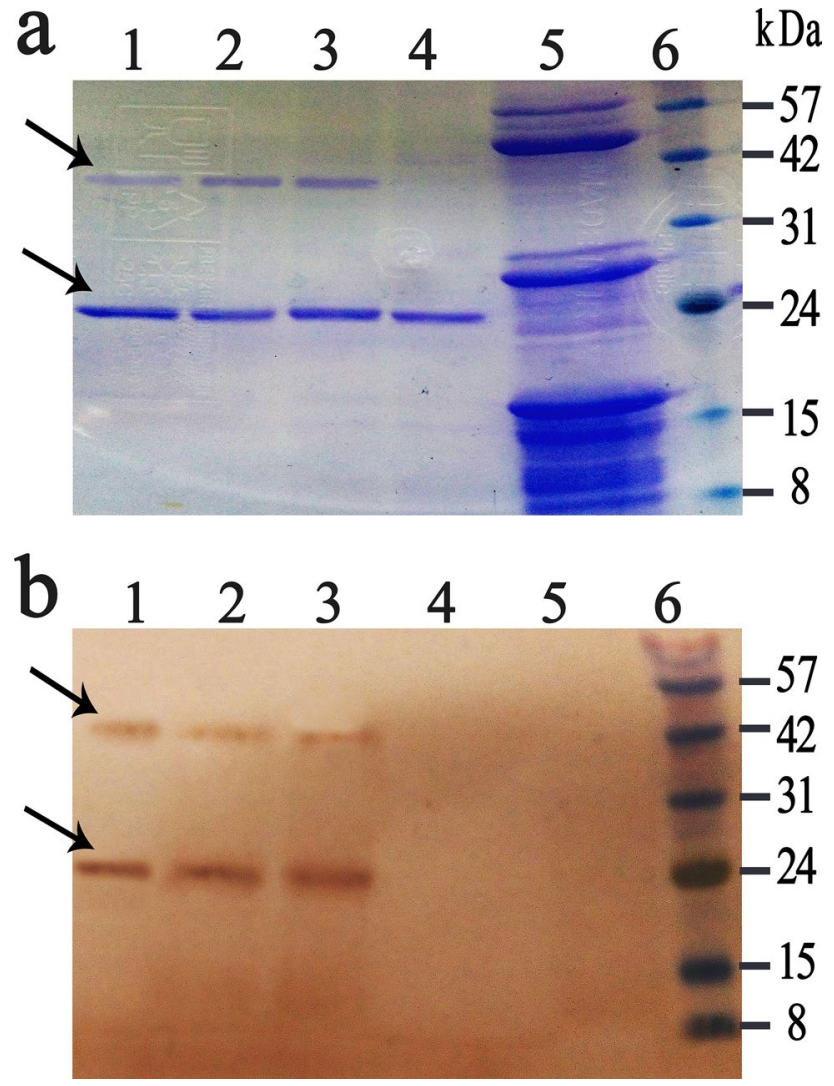

Fig. 6 Analysis of the samples following second step of A2P purification of TSP of co-agroinjected and control leaves. a SDS-PAGE and b western blot; 1,2,3 the extract of Hyd-BMP2 and P19 coagroinjected leaves (the lower phase of step two): 4 the extract of negative control infiltrated leaf (the lower phase of step two): 5 the extract of negative control infiltrated leaf (the upper phase of step two): 6 Sinaclone Protein Molecular Marker

Co1-(AEEEK)3- IL-2-(GGGGS)3- PfCelTOS structure as a fusion protein was suitable to be expressed in edible host cells for oral delivery (Shamriz and Ofoghi 2016). It is a concern with how regular secondary structures compactly pack into the 3D structure of the protein. A previous study reported that $\alpha$ proteins have the highest radius of gyration throughout the protein size range considered, suggesting a less tight packing as compared with $\beta$ - and $(\alpha+\beta)$-proteins. The lowest radius of gyration and, accordingly, the tightest packing are characteristic of $\alpha / \beta$-proteins (Lobanov et al. 2008). Therefore, it will be useful to calculate the radius of gyration for designing a fusion protein.

To have high amount of expression in plant system several strategies designed such as codon optimization and enhancing the transcription and translation. The pTRAkc vector has been used in multiple expression studies due to regulatory sequences such as Scaffold Attachment Regions (SARs) that results in higher amount of transcription. Maclean et al. showed that $11 \%$ of TSP, of tobacco leaves, consisted of L1 protein of Human Papillomavirus type 16

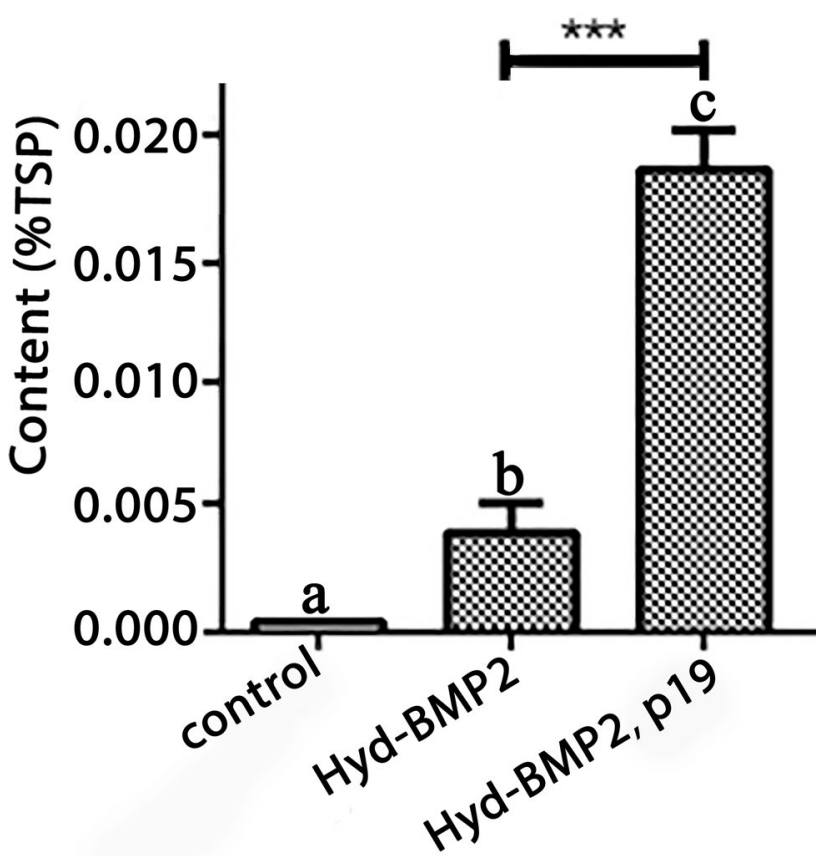

Fig. 7 The result of ELISA test for comparing the expression of recombinant protein expression in co-agroinjected leaves. The * sign indicates a significant difference between groups based on the ANOVA one-way test with $P \leq 0.05$. a-c Indicate the percentage of fusion protein in control plant, plant without P19 construct and plant with P19 construct, respectively

applying pTRAkc vector (Maclean et al. 2007). It is known that the structural features of the 5 'UTR could significantly affect the rate of translation initiation of a gene, moreover, translation efficiency of eukaryotic mRNAs depends on the nucleotide sequence flanking the start codon, namely the start codon context or Kozak sequence (Kozak 1989). The partial sequence of the UAAA CAAUGGCU was used to increase the efficiency of translation initiation as shown before (Mohammadzadeh et al. 2014). Wydro et al. have shown that the appropriate time for the production of recombinant protein in plants is 5 days post agroinjection, which depend on different factors such as age of the leaf (Wydro et al. 2006). In the present study, the agroinjected leaves were left for (5-7 days). However, Ma et al. reported that 4 days post infiltration is the best timing for higher expression in tobacco (Ma et al. 2003). Fast downstream processing is one of the major factors that affect the yield of purified recombinant protein. Fusion with Hydrophobin facilitates the purification process applying A2P.

The A2P is an efficient and economic separation technique. Nouri et al. have reported a 127 percent increase in the partitioning of vanillin using this separation method (Nouri et al. 2019). During A2P, the Triton concentrates the Hydrophobin fusion protein inside micelle structures and partitions them toward the surfactant phase while the 
majority of the proteins remain in the aqueous phase (Linder 2009).

BMP2 has been expressed in different systems as monomer and dimer proteins. In denaturing conditions, BMP2 is in monomeric form, while the native BMP2 is active as a homodimer. (Scheufler et al. 1999). Naturally, 7 cysteines are present in mature BMP2 made up of 114 amino acids. The folding of the monomeric molecule depends on six cysteines, however, the dimer form is due to the seventh cysteine that builds a bridge between monomers (Scheufler et al. 1999); (Hillger et al. 2005). SDS PAGE analysis has shown purification of a TSP protein of all samples that copurifies with monomer band and could be seen as $22-24 \mathrm{kDa}$ (Joensuu et al. 2012), as it could be seen in the upper phase includes other plant proteins with different intensities. In the present study, the result of Western blotting has shown that the plant cells produced Hyd-BMP2 in both monomer and dimer forms which could be easily concentrated and purified by the $\mathrm{A} 2 \mathrm{P}$ technique, however to confirm the characteristics of produced recombinant protein there is a need for further analytical investigations.

Our results showed that human pharmaceutical proteins such as BMP2 could be expressed using transient expression system. Plant transient expression is a fast production system and to ameliorate the purification process and its speed, A2P is a good candidate. Hydrophobin could be fused to the pharmaceutical recombinant protein and later separated using different techniques.

Acknowledgements I would like to express my special thanks to MS. Daeizadeh, for her help and other members of lab for their continued support and encouragements.

\section{References}

Bedrov D, Piquemal J-P, Borodin O, MacKerell AD, Roux B, Schröder C (2019) Molecular dynamics simulations of ionic liquids and electrolytes using polarizable force fields. Chem Rev 119:7940-7995

Berger BW, Sallada ND (2019) Hydrophobins: multifunctional biosurfactants for interface engineering. J Biol Eng 13:10-10

Bhargava BL, Balasubramanian S (2005) Dynamics in a room-temperature ionic liquid: a computer simulation study of 1,3-dimethylimidazolium chloride. J Chem Phys 123:144505

Chen H, Nelson RS, Sherwood JL (1994) Enhanced recovery of transformants of Agrobacterium tumefaciens after freeze-thaw transformation and drug selection. Biotechniques 16(664-8):70

Childers MC, Daggett V (2018) Validating molecular dynamics simulations against experimental observables in light of underlying conformational ensembles. J Phys Chem B 122:6673-6689

Dehghani B, Mousavi A, Hasannia S, Salmanian AH, Sharafi A, Eftekhariyan Ghamsari mr (2018) Expression of recombinant human bone morphogenetic protein 2 (rhBMP2) in tobacco transgenic hairy roots. J Agric Biotechn 10:21-34

Gamer LW, Pregizer S, Gamer J, Feigenson M, Ionescu A, Li Q, Han $\mathrm{L}$, Rosen V (2018) The role of Bmp2 in the maturation and maintenance of the murine knee joint. J Bone Miner Res 33:1708-1717
Gao Y, Suo GL, Han J, He ZQ, Yao W, Dai JW (2006) Expression of human BMP-2 gene in different tissues of tobacco plants. Yi Chuan Xue Bao 33:56-62

Hakanpää J, Szilvay GR, Kaljunen H, Maksimainen M, Linder M, Rouvinen J (2006) Two crystal structures of Trichoderma reesei hydrophobin HFBI-the structure of a protein amphiphile with and without detergent interaction. Protein Sci: Publ Protein Soc $15: 2129-2140$

Hillger F, Herr G, Rudolph R, Schwarz E (2005) Biophysical comparison of BMP-2, ProBMP-2, and the free pro-peptide reveals stabilization of the pro-peptide by the mature growth factor*. J Biol Chem 280:14974-14980

Holmberg K (2003) Surfactants and polymers in aqueous solution. Chichester, Wiley

Imani-Fooladi AA, Yousefi F, Mousavi SF, Amani J (2014) In silico design and analysis of TGF $\alpha$ L3-SEB fusion protein as 'a New Antitumor Agent' candidate by ligand-targeted superantigens technique. Iran J Cancer Prev 7:152-164

Inai K, Norris RA, Hoffman S, Markwald RR, Sugi Y (2008) BMP-2 induces cell migration and periostin expression during atrioventricular valvulogenesis. Dev Biol 315:383-396

Israel DI, Nove J, Kerns KM, Moutsatsos IK, Kaufman RJ (1992) Expression and characterization of bone morphogenetic protein-2 in Chinese hamster ovary cells. Growth Factors 7:139-150

Joensuu JJ, Conley AJ, Lienemann M, Brandle JE, Linder MB, Menassa R (2010) Hydrophobin fusions for high-level transient protein expression and purification in Nicotiana benthamiana. Plant Physiol 152:622-633

Joensuu JJ, Conley AJ, Linder MB, Menassa R (2012) Bioseparation of recombinant proteins from plant extract with hydrophobin fusion technology. Methods Mol Biol 824:527-534

Kapila J, De Rycke R, Van Montagu M, Angenon G (1997) An Agrobacterium-mediated transient gene expression system for intact leaves. Plant Sci 122:101-108

Khalesi M, Deckers SM, Gebruers K, Vissers L, Verachtert H, Derdelinckx G (2012) Hydrophobins: exceptional proteins for many applications in brewery environment and other bio-industries. Cerevisia 37:3-9

Kirsch T, Sebald W, Dreyer MK (2000) Crystal structure of the BMP2-BRIA ectodomain complex. Nat Struct Biol 7:492-496

Kohagen M, Mason PE, Jungwirth P (2016) Accounting for electronic polarization effects in aqueous sodium chloride via molecular dynamics aided by neutron scattering. J Phys Chem B 120:1454-1460

Kozak M (1989) The scanning model for translation: an update. J Cell Biol 108:229-241

Kurppa K, Reuter LJ, Ritala A, Linder MB, Joensuu JJ (2018) Insolution antibody harvesting with a plant-produced hydrophobinprotein A fusion. Plant Biotechnol J 16:404-414

Lemkul JA, Roux B, van der Spoel D, MacKerell AD Jr (2015) Implementation of extended Lagrangian dynamics in GROMACS for polarizable simulations using the classical Drude oscillator model. J Comput Chem 36:1473-1479

Lemkul JA, Huang J, Roux B, MacKerell AD (2016) An empirical polarizable force field based on the classical Drude oscillator model: development history and recent applications. Chem Rev 116:4983-5013

Linder MB (2009) Hydrophobins: proteins that self assemble at interfaces. Curr Opin Colloid Interface Sci 14:356-363

Lobanov MY, Bogatyreva NS, Galzitskaya OV (2008) Radius of gyration as an indicator of protein structure compactness. Mol Biol 42:623-628

Ma JK, Drake PM, Christou P (2003) The production of recombinant pharmaceutical proteins in plants. Nat Rev Genet 4:794-805

MacKerell AD, Karplus M (1991) Importance of attractive van der Waals contribution in empirical energy function 
models for the heat of vaporization of polar liquids. J Phys Chem 95:10559-10560

Maclean J, Koekemoer M, Olivier AJ, Stewart D, Hitzeroth II, Rademacher T, Fischer R, Williamson AL, Rybicki EP (2007) Optimization of human papillomavirus type 16 (HPV-16) L1 expression in plants: comparison of the suitability of different HPV-16 L1 gene variants and different cell-compartment localization. J Gen Virol 88:1460-1469

Merlin M, Gecchele E, Capaldi S, Pezzotti M, Avesani L (2014) Comparative evaluation of recombinant protein production in different biofactories: the green perspective. Biomed Res Int 2014:136419

Miyata KN, Nast CC, Dai T, Dukkipati R, LaPage JA, Troost JP, Schurgers LJ, Kretzler M, Adler SG (2018) Renal matrix Gla protein expression increases progressively with CKD and predicts renal outcome. Exp Mol Pathol 105:120-129

Mohammadzadeh S, Khabiri A, Roohvand F, Memarnejadian A, Salmanian AH, Ajdary S, Ehsani P (2014) Enhanced-transient expression of hepatitis $\mathrm{C}$ virus core protein in nicotiana tabacum, a protein with potential clinical applications. Hepat Mon 14:e20524

Nocito D, Beran GJO (2018) Massively parallel implementation of divide-and-conquer jacobi iterations using particle-mesh ewald for force field polarization. J Chem Theory Comput 14:3633-3642

Nouri M, Shahriari S, Pazuki G (2019) Increase of vanillin partitioning using aqueous two phase system with promising nanoparticles. Sci Rep 9:19665

Ren P, Ponder JW (2004) Temperature and pressure dependence of the AMOEBA water model. J Phys Chem B 108:13427-13437

Sambrook J (2001) Molecular cloning : a laboratory manual, 3rd edn. Cold Spring Harbor Laboratory Press, Cold Spring Harbor, N.Y (C2001)

Scheufler C, Sebald W, Hülsmeyer M (1999) Crystal structure of human bone morphogenetic protein-2 at 2.7 a resolution. $\mathrm{J}$ Mol Biol 287:103-115

Shamriz S, Ofoghi H (2016) Design, structure prediction and molecular dynamics simulation of a fusion construct containing malaria preerythrocytic vaccine candidate, PfCelTOS, and human interleukin 2 as adjuvant. BMC Bioinformatics 17:71
Sivars U, Tjerneld F (2000) Mechanisms of phase behaviour and protein partitioning in detergent/polymer aqueous two-phase systems for purification of integral membrane proteins. Biochim Biophys Acta 1474:133-146

Tiwari S, Verma PC, Singh PK, Tuli R (2009) Plants as bioreactors for the production of vaccine antigens. Biotechnol Adv 27:449-467

Tsuda H, Wada T, Ito Y, Uchida H, Dehari H, Nakamura K, Sasaki K, Kobune M, Yamashita T, Hamada H (2003) Efficient BMP2 gene transfer and bone formation of mesenchymal stem cells by a fiber-mutant adenoviral vector. Mol Ther 7:354-365

Vallejo LF, Brokelmann M, Marten S, Trappe S, Cabrera-Crespo J, Hoffmann A, Gross G, Weich HA, Rinas U (2002) Renaturation and purification of bone morphogenetic protein-2 produced as inclusion bodies in high-cell-density cultures of recombinant Escherichia coli. J Biotechnol 94:185-194

Weber D, Kotzsch A, Nickel J, Harth S, Seher A, Mueller U, Sebald W, Mueller TD (2007) A silent H-bond can be mutationally activated for high-affinity interaction of BMP-2 and activin type IIB receptor. BMC Struct Biol 7:6

Wydro M, Kozubek E, Lehmann P (2006) Optimization of transient Agrobacterium-mediated gene expression system in leaves of Nicotiana benthamiana. Acta Biochim Pol 53:289-298

Yeh I-C, Hummer G (2004) System-size dependence of diffusion coefficients and viscosities from molecular dynamics simulations with periodic boundary conditions. J Phys Chem B 108:15873-15879

Zhang H, Wu J, Zhang Y, Fu N, Wang J, Zhao S (2010) Optimized procedure for expression and renaturation of recombinant human bone morphogenetic protein- 2 at high protein concentrations. Mol Biol Rep 37:3089-3095

Zhang Y, Liu Y, Wildsoet CF (2012) Bidirectional, optical signdependent regulation of BMP2 gene expression in chick retinal pigment epithelium. Invest Ophthalmol vis Sci 53:6072-6080

Publisher's Note Springer Nature remains neutral with regard to jurisdictional claims in published maps and institutional affiliations. 